\title{
Virtual fractional flow reserve and virtual coronary stent guided percutaneous coronary intervention
}

\author{
Fang-Yang Huang ${ }^{1}$, Qi Liu ${ }^{1}$, Xiao-Xiao $\mathrm{Liu}^{2}$, Bin $\mathrm{Ma}^{2}$, Ye $\mathrm{Zhu}^{1}$ \\ ${ }^{1}$ Department of Cardiology, West China Hospital, Sichuan University, Chengdu, China \\ ${ }^{2}$ Shenzhen Keya Medical Technology Corporation, Shenzhen, China
}

Deep vessel (DV)-angio is a newly developed software utilizing machine learning methods to calculate virtual fractional flow reserve (vFFR) based upon coronary angiogram (CAG). Briefly, a supervised deep learning and neural net model is trained in advance from hundreds of cases to map the extracted three-dimensional vascular structures and the corresponding blood flow estimation. Furthermore herein, a virtual coronary stent (VCS) technology has been developed that simulates post-VCS coronary artery geometries and estimates corresponding vFFR.

A 70-year-old male patient with recurrent chest pain was scheduled to undergo CAG. CAG revealed a $90 \%$ luminal stenosis in distal right coronary artery (RCA) after intracoronary administration of nitrates (Fig. 1A). The left coronary artery is normal. Two orthogonal CAG cines were imported into the DV-angio that reconstructed the RCA as in Figure 1B. The vFFR was 0.78 in the distal RCA while an invasive FFR measured with the pressure wire was 0.75 (Fig. 1C). Then, the DV-angio modeled the targeted coronary artery after a simulated $3.5-\mathrm{mm} \times 24-\mathrm{mm}$ stent was implanted, and estimated the post-VCS FFR to be 0.97 (Fig. 1D). These processes took about $4 \mathrm{~min}$. After implanting the same stent as the simulated one at the stenosis site (Fig. 1E), the actual FFR was improved to 0.99 (Fig. $1 \mathrm{~F}$ ). The patient no longer experienced angina pectoris during follow-up.

The feasibility of VCS-guided percutaneous coronary intervention (PCI) was first reported herein, based on machine learning methods in a real-world case. As CAG allows ad hoc PCI after strategy determined, the CAG-based vFFR and VCS will have a great practical application foreground.

\section{Fundings}

The present study received grants from the National Key R\&D Program of China (2017YFC0910004 and 2017YFB0702500), the National Natural Science Foundation of China (grant number: 81900258), the Sichuan Science and Technology Program (2020YFS0246), the China Postdoctoral Science Foundation Grant (2019M663521), the Post-Doctor Research Project, Sichuan University (2020SCU12029), and the PostDoctor Research Project, West China Hospital, Sichuan University (2019HXBH016).

Conflict of interest: None declared

Address for correspondence: Ye Zhu, MD, Department of Cardiology, West China Hospital, Sichuan University, 37 Guoxue Street, Chengdu 610041, Sichuan, China, tel: +86-021-85422343, e-mail: zhuye1974@163.com 


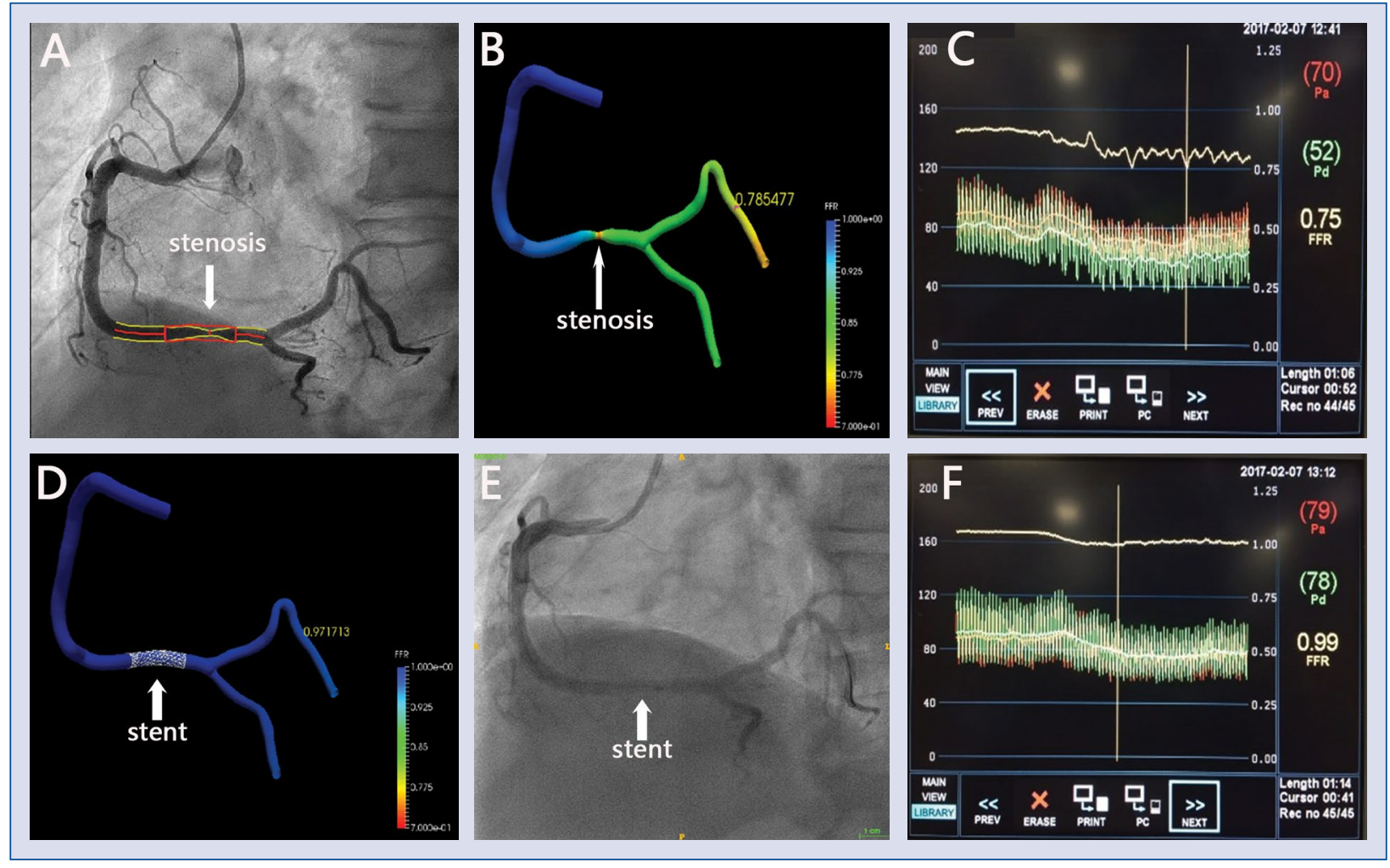

Figure 1. A. The right coronary angiogram was displayed; B. Deep vessel (DV)-angio reconstructed three-dimensional right coronary artery. A virtual fractional flow reserve (FFR) is estimated to be 0.78 ; $\mathbf{C}$. The actual FFR was measured by pressure wire; $\mathbf{D}$. The predicted DV-angio FFR, after a simulated $3.5-\mathrm{mm} \times 24-\mathrm{mm}$ stent was implanted, was 0.97 ; E. The right coronary angiogram was displayed after implantation of a $3.5-\mathrm{mm} \times 24-\mathrm{mm}$ stent; F. The true wire poststent FFR was 0.99 . 